\title{
Towards Palm-Size Autonomous Helicopters
}

\section{Journal Article}

\section{Author(s):}

Bouabdallah, Samir; Bermes, Christian; Grzonka, Slawomir; Gimkiewicz, Christiane; Brenzikofer, Alain; Hahn, Robert; Schafroth, Dario; Grisetti, Giorgio; Burgard, Wolfram; Siegwart, Roland

\section{Publication date:}

2011-01

Permanent link:

https://doi.org/10.3929/ethz-b-000030902

\section{Rights / license:}

In Copyright - Non-Commercial Use Permitted

\section{Originally published in:}

Journal of Intelligent \& Robotic Systems 61(1-4), https://doi.org/10.1007/s10846-010-9483-y 


\title{
Towards Palm-Size Autonomous Helicopters
}

\author{
Samir Bouabdallah • Christian Bermes • Slawomir Grzonka • \\ Christiane Gimkiewicz • Alain Brenzikofer • Robert Hahn • \\ Dario Schafroth • Giorgio Grisetti · Wolfram Burgard · Roland Siegwart
}

Received: 1 February 2010 / Accepted: 1 September 2010 / Published online: 2 December 2010

(c) Springer Science+Business Media B.V. 2010

\begin{abstract}
Fly EU project started in 2006 with the idea to build an autonomous micro helicopter, comparable in size and weight to a small bird. Several scientific and
\end{abstract}

S. Bouabdallah $(\varangle) \cdot$ C. Bermes $\cdot$ D. Schafroth

Autonomous System Lab, Swiss Federal Institute of Technology Zurich (ETHZ),

CLA E16.2, Zurich, Switzerland

e-mail: samir.bouabdallah@mavt.ethz.ch

S. Grzonka · G. Grisetti

Department of Computer Science, Autonomous Intelligent Systems, University of Freiburg,

Georges-Koehler-Allee 079, 79110 Freiburg, Germany

S. Grzonka

e-mail: grzonka@informatik.uni-freiburg.de

G. Grisetti

e-mail: grisetti@informatik.uni-freiburg.de

C. Gimkiewicz

CSEM Zurich Center, Technoparkstrasse 1, 8005 Zürich, Switzerland

A. Brenzikofer

CSEM SA, Technoparkstr. 1, 8005 Zürich, Switzerland

R. Hahn

Fraunhofer-Institut Zuverlaessigkeit und Mikrointegration (IZM), High Density Interconnect \& Wafer Level Packaging Gustav-Meyer-Allee 25, 13355 Berlin, Germany

e-mail: Robert.Hahn@izm.fraunhofer.de

W. Burgard

Institut für Informatik, Faculty for Applied Sciences, Albert-Ludwigs-Universität Freiburg,

Georges-Köhler-Allee, Geb. 079, 79110 Freiburg, Germany

R. Siegwart

ETH Zürich, Inst.f. Robotik u. Intelligente Systeme, HG F 57 Rämistrasse 101,

8092 Zürich, Switzerland

e-mail: rsiegwart@ethz.ch 
technological objectives were identified. This spanned from system-level integration, high efficiency micro-actuation, highly integrated micro vision sensors and IMUs and also low processing power navigation algorithms. This paper shows how most of these objectives were reached, describing the approach and the role of each partner during the whole project. The paper describes also the technological developments achieved like the $80 \mathrm{~g}, 17 \mathrm{~cm}$ micro robotic-helicopter, the $8 \mathrm{~g}$ omnidirectional and steady-state laser scanner, the uIMU, the highly efficient micro motors, the high power-density fuel-cell and the successful graph-based navigation algorithms.

Keywords Micro helicopter • Multi-directional triangulation • Micro actuators • Graph based SLAM

\section{Introduction}

Research on autonomous micro-helicopters has recently made a so important progress, that the research focus shifted from vehicle stabilization to autonomous navigation, which became possible with the newly available sensors and embedded computers. However, the problem starts to be much more complicated if one wants to go down with the scale, let's say below $20 \mathrm{~cm}$. A scale at which off-the-shelf sensors, actuators and computer modules are still bulky and too heavy. Even if there was an important effort put into the development of micro-flying robots, there is no helicopter (below $20 \mathrm{~cm}$ ) that combines reasonable endurance with a decent payload, and all of them are still far from autonomous navigation in narrow indoor environments. The aim of the muFly project was to develop an autonomous micro helicopter comparable in size and weight to a small bird. This challenging goal implies the combination of a large variety of technologies, from aerodynamics up to control and navigation problems. Especially when it comes to very lightweight and small autonomous aerial vehicles, numerous challenges have to be addressed and technical limitations have to be overcome. This paper presents the muFly project and tells what the consortium produced as results in term of science and technology. The key objectives of the project include innovative concepts for power sources, sensors, actuators, navigation and helicopter design and their integration into a very compact system. The project envisaged at the beginning, a complete system weighing about $30 \mathrm{~g}$ and measuring only $10 \mathrm{~cm}$ in diameter, providing the following innovations:

- System level design and optimization of autonomous micro aerial vehicles,

- Multi-functional use of components,

- Design of "smart" miniature inertial sensors and omnidirectional vision sensors with polar pixel arrangement,

- Miniaturized fuel-cells,

- Miniaturized piezoelectric actuators with enhanced power to weight ratios,

- Control and navigation concepts that can cope with limited sensor and processing performance.

The final system was expected to find applications in surveillance of buildings and large indoor areas that are difficult to access on wheels or legs, rescue missions in buildings after natural disasters or terror attacks, surveillance of dangerous areas, chemical and nuclear plants or law enforcement in public areas. 
The muFly consortium was composed of six partners, each one enriching the project with a specific competence. The Swiss Federal Institute of Technology in Zürich (ETHZ), was responsible for the system design and integration, modeling and control, and aerodynamics optimization at low Reynolds number. The "Centre Suisse d'Electronique et de Microtechnique SA" (CSEM-Zürich) was a key partner responsible for designing a customized micro camera sensor and its optics. The well known company XSENS was entrusted the design of a micro IMU, adapted to the constraints of muFly. The French company CEDRAT active in the market of micro piezo-electric actuators, was in charge of designing the micro-motors of muFly helicopter. The Technical University of Berlin (TUB) was responsible for providing the power source (fuel-cell or battery). Finally, the Albert-Ludwigs-University of Freiburg in Germany (ALUFR) was a key partner in charge of the autonomous navigation system.

\subsection{The Challenges}

The challenges facing MAV development are numerous, a good survey of these challenges is presented in [1]. They can be summarized as:

- The lack of accurate models of flow separation and unsteady aerodynamics at low Reynolds numbers.

- The low efficiency of propulsion systems at small scales.

- The lack of adapted structures and materials.

- The requirement for too much processing power and high resolution sensors for stabilization and navigation algorithms.

- The low capacity of actual energy storage devices.

- The lack of powerful methodologies for system level integration which remains a key challenge.

\subsection{The Possible Solutions}

In order to approach the goal of the muFly project, the team proposes a list of possibilities to alleviate some limitations on MAV design:

- Enhance propeller efficiency by acting on different parameters like: tip shape and leading edge as well as exploiting the Coanda effect or Gurney flaps.

- Enhance motor efficiency by optimizing micro brushless outrunner motors.

- Use of multi-functional components made of lightweight composite material.

- Use of low computational-cost navigation algorithms.

- Use of a fuel-cell power source.

- Optimize the overall system.

\subsection{State of the Art}

Drawing-up the state-of-the-art and considering only micro helicopters at a scale comparable to a small bird, is a quick job. In fact, only few research teams are active in this area. One interesting project targeting a similar size but based on a quadrotor concept is the Mesicopter developed at Stanford University [2]. The result 
of this project is a wired micro-quadrotor performing an axially constrained take-off. Another development from EPSON (http://www.epson.co.jp/) is a $13.6 \mathrm{~cm}$ microhelicopter able to hover $3 \mathrm{~min}$. It is remotely operated via a Bluetooth link and can carry an onboard camera. The Prox Dynamics PD-100 Black Hornet is one of the most important developments in the field. It targets a fully autonomous nanohelicopter weighing about $15 \mathrm{~g}$ and able to carry a micro camera and fly outdoors. Other types of micro-flying machines make use of fixed [3] or flapping wings (Aerovironment, http://www.aerovironment.com, http://www.delfly.nl). Whereas, the fixed wings concepts are well adapted and developed for autonomous flight in free space (outdoor), the miniaturized flapping wing concepts are still far from high autonomy, energy and navigation wise. The main reason for this somewhat disappointing status is that most projects did not address system level optimization appropriately and did not assemble all the key technologies in a single project. Additionally, the required technology needed for the successful design and integration of a fully autonomous micro air vehicle became available only very recently and has still to be adapted and optimized through an integral design effort. However, we are still facing stringent technological limitations in power storage devices, integrated sensors, miniature actuators and processing power.

\section{Understanding the Problematic}

Before starting the design of the first prototype of muFly, it was necessary to investigate some open questions. This concerned primarily the aerodynamics related to small rotors arranged in a coaxial configuration. It concerned also the understanding and the formulation of the behavior of the stabilizer bar. There was also a practical problem related to the way to test the stability of the MAV in a repeatable and safe way. These issues motivated the design of three different test-benches, which allow repeatable measurements in a lab-like environment.

\subsection{Rotor Test-Bench}

The aerodynamics at low Reynolds number represent one of the strongest challenges facing future MAV development, see [1]. In fact, the power needed for the propulsion of a VTOL MAV represents about $90 \%$ of the total power consumption. Beside reducing the mass the improvement of the propulsion group is one of the most important tasks to increase the autonomous flight time. The aerodynamics in this small scale are very different from full scale helicopter: The Reynolds number $R e$ is extremely low (below 60,000), which leads to a strong influence of viscous effects. Phenomena like laminar separation bubbles strongly affect the aerodynamic efficiency which is much lower than in full scale. This problem was tackled in muFly from both theoretical and experimental points of view. Since only few data sets are available in literature, we decided to build our own test-bench in order to: on the one hand collect experimental data and from the other hand validate simulation results. The idea was to build a test bench for thrust and torque measurement. The setup is a coaxial rotor system with a thrust sensor and a torque sensor integrated to measure the force and torque in the rotor shaft direction. Figure 1 shows the rotor 
Fig. 1 Coaxial rotor test-bench. The rotor blades are produced on an Object 3D printer, with high resolution. Different airfoils and shapes can be easily tested

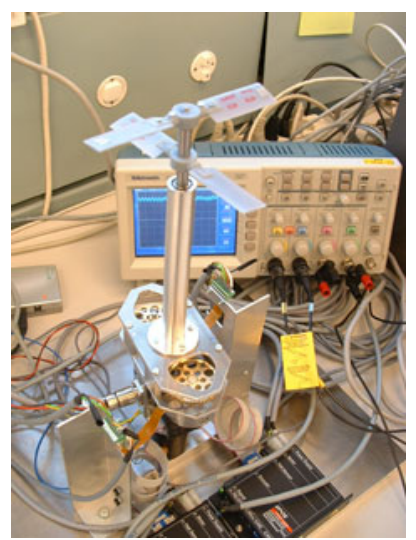

test-bench. The test-bench is interfaced to Matlab for easy control and analysis of the data.

\subsection{Stabilizer Test-Bench}

The stabilizer test-bench is an improved version of the test-bench presented in [4]. The core idea of the system is to mount a complete coaxial rotor setup with a passive lower rotor and an upper rotor augmented by a stabilizer bar on a six axis load cell. Thus the rotor forces and moments can be measured, and the effect of mounting a stabilizer bar, as well as the influence of stabilizer bar design variations can be quantified. The specific goal of the test-bench measurements is to identify the stabilizer bar following time and phase angle for the muFly rotor system. The testbench is designed such that the key design parameters of the stabilizer bar, namely phase angle $\alpha$ with respect to the blade pitching axis, and flapping inertia of the stabilizer bar $I_{\mathrm{sb}}$ can be varied. The rotors can be driven at different speeds, and for verification purposes the lower passive rotor can be fully shut down, leading to a single rotor test setup. The schematic layout of the setup is shown in Fig. 2 (left).
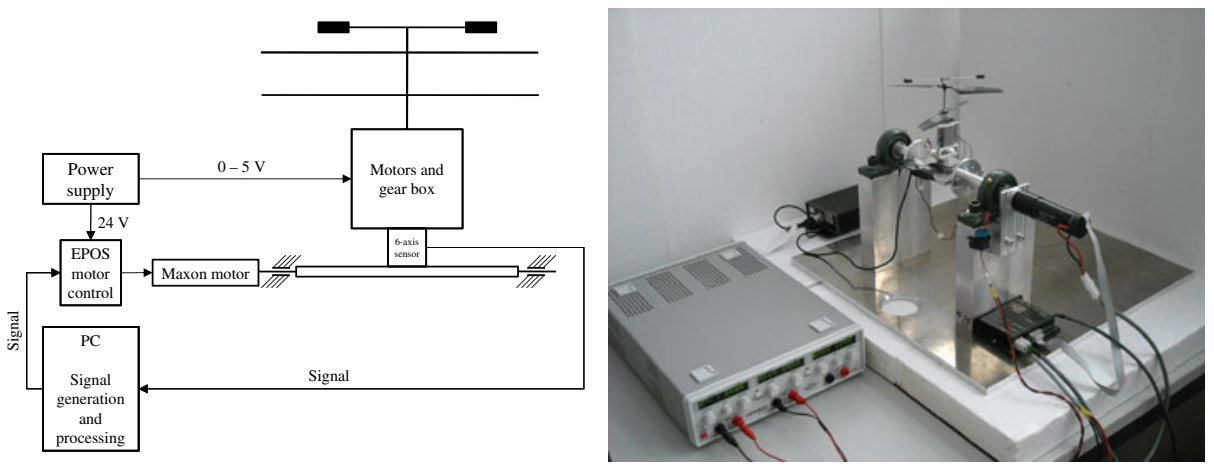

Fig. 2 Schematic layout of the stabilizer test-bench (left), and photo of the test-bench at the ASL (right). A lab power supply is used for powering the motors and the electronics 
The coaxial rotor with stabilizer bar are held by a motor and gearbox housing, which is mounted on the six axis load sensor. The two coreless DC motors can be driven with up to $5 \mathrm{~V}$ from a lab power supply. The rotors and load cell are fixed on a metal plate, which can be tilted around one horizontal axis by a Maxon motor. This motor is controlled with an EPOS motor controller, receiving its control input from a signal generation program running on the PC. The analog data from the load cell measurements is digitalized at a sampling rate of $10 \mathrm{kHz}$ and sent to the computer for storage and digital signal processing. A photo of the test-bench with its major components is shown in Fig. 2 (right). The tilting platform with the Maxon motor are supported by two heavy ball bearings, which are mounted on a rigid aluminum structure and base plate. This construction minimizes the play and flexibility in the test-bench and results in higher precision of the measurements. Figure 3 shows the input signals to the tilting platform for the following time experiments (left) and the phase angle measurements (right). While the ramp input is more suited for identifying the stabilizer bar's following time $T_{\mathrm{f}}$, the sinusoidal input is better to measure phase differences between signals from different stabilizer phase angles. The detailed results concerning stabilizer-bar following time and offset angle are thoroughly presented in [5] (to appear).

\subsection{Dynamics Test-Bench}

In order to ensure a stable flight behavior a correct control is necessary. For the system identification as well as for the first flights with a new controller, the helicopter needs a safe environment in which full motion is possible and crashes are avoided. To study the behavior of a helicopter, the recording of the flying path which includes information about the position and the orientation is also needed. From these needs, came the idea to design a cable-based robot. The test bench has to permit full motion ( $6 \mathrm{DoF})$, measure the position and orientation of the micro helicopter. Its second function is to provide a safe environment for the robots first flights, so that crashes can be avoided in situations where the rotorcraft gets out of control. Furthermore, the test-bench is expected to compensate for its own mass since the muFly is very lightweight. The helicopter would then imply motion on the
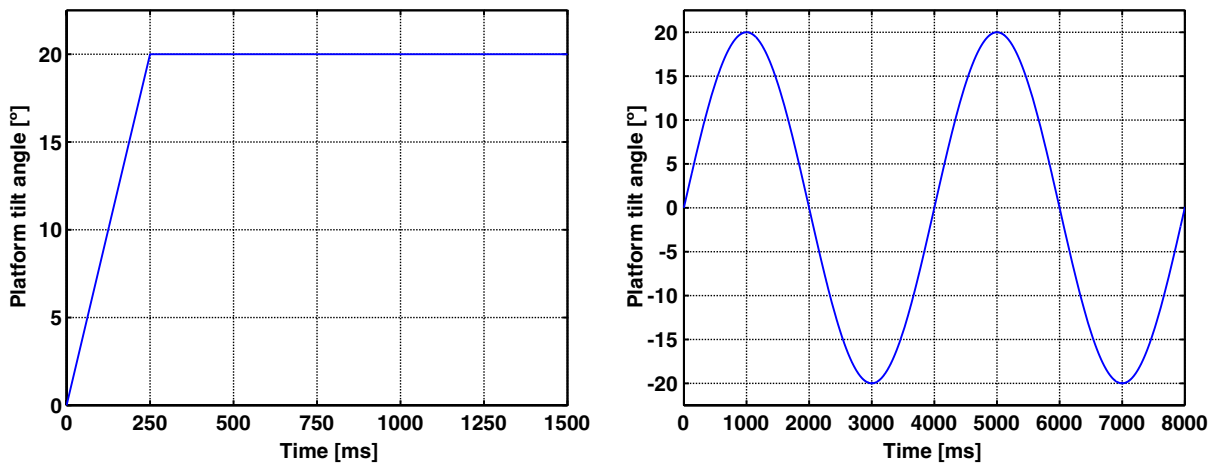

Fig. 3 Tilting platform input signals for the following time experiments (left) and the phase angle measurements (right) 
test-bench, but not sustain any motion from it. The dynamical forces from the test bench should act on the center of gravity $(\mathrm{CoG})$ of the micro helicopter. Unfortunately, the $\mathrm{CoG}$ is not accessible and thus kinematic equivalents to a spherical joint mounted in the $\mathrm{CoG}$ must be found. The $\mathrm{CoG}$ of the helicopter is on its central axis. Moreover, the forces acting on the moving helicopter due to inertia and friction in the test bench should be kept far smaller than the weight of the rotorcraft $(80 \mathrm{~g})$. Concerning the working volume, the helicopter needs to be able to move at least $150 \mathrm{~mm}$ in each direction, which leads to a spherical workspace with a diameter of $300 \mathrm{~mm}$. The rotations about the $X$ - and $Y$-axis (roll and pitch) of the helicopter have to be possible within a range of $\pm 15^{\circ}$. There should preferably be no limitation on the rotation about the $Z$-axis (yaw), but minimum $\pm 180^{\circ}$. should be allowed. The largest dimension of the test stand should not exceed $1.5 \mathrm{~m}$ for proper use in a laboratory. The output of the measurement of position and orientation will not be used for controlling purposes as the helicopter is meant to be autonomous and therefore has its own sensors for measuring its state. The measurement system is proposed to record the flying-path of the rotorcraft. It needs to be only fast enough to record the movements properly, this means the frequency should not be less than $30 \mathrm{~Hz}$. There are in fact few existing solutions. A literature study was performed in scientific online databases in order to find test stands used in other academic projects. A web research using popular search engines resulted mostly in flying stands for training with remote control model helicopters. There are for instance: Whirlingarm concepts (max $5 \mathrm{DoF}$ ) [6], rotation test stands (max $3 \mathrm{DoF}$ ) [7], test-benches with parallel kinematics (max 6 DoF) [8], test-bench with serial kinematics (max 6 DoF) [9]. A prototype of the final design is shown in Fig. 4. This concept compensates the gravity effects actively with three controlled motors mounted at the frame. To provide full gravity compensation also for the rotational degrees of freedom, the center of mass of each of the three arms has to be aligned with the center of mass of the helicopter. To balance every main arm of the central structure with respect to the center point, a second arm on the opposite side of the main axis with a counterweight on its end has to be added. Included in the balancing of each arm is one third of the mass of the main pivot and the coupling. The three arms are sharing the mass of the

Fig. 4 The muFly vehicle test-bench. The central structure is supported by three actively controlled lines

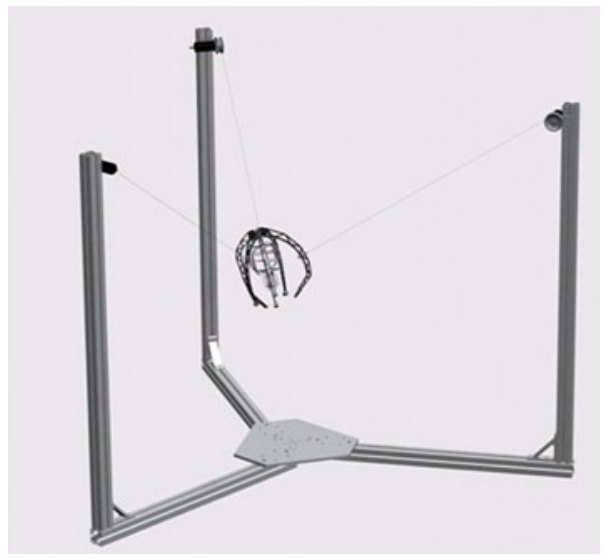


pivot and the coupling for compensation. The mass of the rollers with bearings and hangers do not need to be balanced.

\section{The muFly Prototypes}

In this section, two prototypes of the muFly helicopter, which have been built and flown, are introduced. Special attention is paid to the integration problems that have to be solved for each of them. The section finishes with a mass comparison of the two prototypes.

\subsection{First Prototype}

The prototype 1 of muFly is developed as a general test platform. It allows for inflight testing of system hardware and the attitude and altitude control algorithms for the helicopter [10]. The complete and assembled system is shown in Fig. 5. The design goal of this prototype is to provide a robust platform with a low degree of integration and a high level of modularity to allow for extensive testing in changing configurations. Due to the low level of integration, components can be easily exchanged for maintenance and repair, and new components can be added with only small modifications. The main design constraints to be incorporated in the prototype are the following:

- Integration of the relatively heavy $(11 \mathrm{~g})$ and bulky $(48 \mathrm{~mm} \times 33 \mathrm{~mm} \times 15 \mathrm{~mm})$ standard Inertial Measurement Unit (IMU) MTi OEM produced by Xsens.

- Free field of view for the ultrasonic rangefinder to measure the ground distance of the helicopter.

To meet these constraints, the helicopter is basically designed in two functional sections, which are a propulsion/drivetrain section and an electronics/sensor section. The propulsion/drivetrain section consists of a model helicopter drive train and rotor

Fig. 5 Assembled muFly 1 prototype. Modular and robust, tt allowed testing the preliminary control algorithms

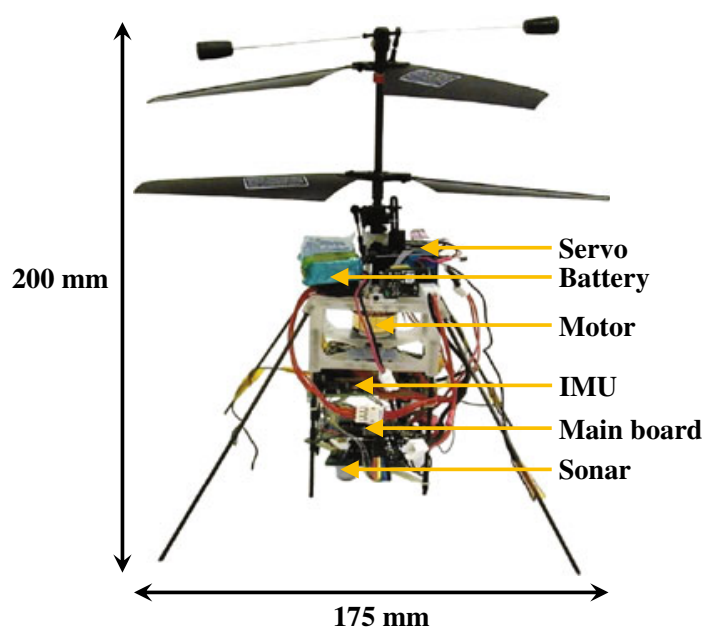


system taken from Walkera's 5G6 coaxial helicopter including the servo motors of type WK-03-01. The rotors are driven by two brushless direct current (BLDC) outrunner motors LRK 13-4-15Y, using 1:1.5 ratio spur gears. All these components are retained by a central structural part, which also serves as the connection to the sensor/electronics section and holds the landing gear. This central frame is produced in rapid prototyping and shown as a CAD model in Fig. 6. The advantage of using stereolithography rapid prototyping lies in the realizability of very complex three dimensional structures at a relatively low cost for small quantities. Moreover, the structure can be easily modified and manufactured within about $3 \mathrm{~h}$. On the downside, however, the properties of the rapid prototyping material are relatively poor. The material becomes slightly brittle with increasing age, and its E-modulus is reduced by the temperature increase due to motor waste heat. Therefore, the main structure is certainly not an optimum in mass, but a good compromise for a test platform that needs high adaptability to changing system components. The electronics/sensor section consists of four carbon fiber rods in a square arrangement, which are pushed into receptacles at the bottom of the main frame and serve as a rack for all electronic components and sensors. Hence, the components can be pushed onto the rods and stacked. This allows for fixation of the IMU with dedicated adapters, as well as fixation of the main processing board, the motor controllers and the ultrasonic rangefinder at the bottom of the helicopter for a free field of view. This is also shown in Fig. 5. A complete overview of the mass contributions of the individual components to the helicopter's total mass of $95.84 \mathrm{~g}$ is given in Table 1. Inspection of Fig. 5 and the quantification in Table 1 clearly show that the modularity and interchangeability of the helicopter come at the price of an increased total mass. The need for various cables and connectors, and the fact that the helicopter's structural components have to be tailored to the electronic components and sensors, lead to large mass contributions of the helicopter's structure and miscellaneous electronics, which mostly summarizes cables, connectors and additional components that cannot be integrated into the main electronic board. Also, with a mass of $11 \mathrm{~g}$, the IMU contributes more then $10 \%$ to the total helicopter mass. Since for the rotors and propulsion system used the maximum take-off mass lies at $100 \mathrm{~g}$, the helicopter is fully loaded in this configuration and cannot carry further components, for instance the $x$-y-position sensor that is required for full position control. Being

Fig. 6 Central frame as CAD model with receptacles for the electronics/sensor section and the landing gear

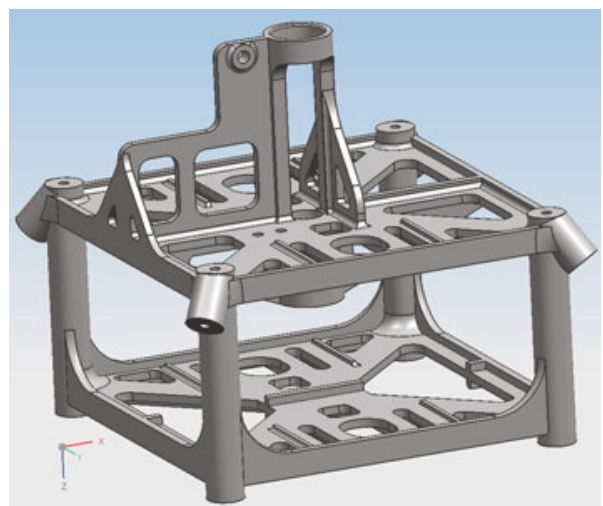


Table 1 Mass distribution of the muFly 1 prototype

\begin{tabular}{llclc}
\hline & Component & $\begin{array}{c}\text { Comp. } \\
\text { mass }(\mathrm{g})\end{array}$ & $\begin{array}{l}\text { Quant. } \\
(-)\end{array}$ & $\begin{array}{c}\text { Total } \\
\text { mass }(\mathrm{g})\end{array}$ \\
\hline Structure & Main frame & 7.83 & 1 & 7.83 \\
& Carbon rod & 0.44 & 4 & 1.76 \\
& Motor holder & 0.35 & 4 & 1.40 \\
& Misc. & 5.27 & 1 & 5.27 \\
& Total mass structure & & & 16.26 \\
Sensors & 11.00 & 1 & 11.00 \\
& IMU & 3.75 & 1 & 3.75 \\
& Ultrasonic rangefinder & & 14.75 \\
Electronics & Total mass sensors & & & 4.40 \\
& Motor contr. & 2.20 & 2 & 8.30 \\
& Main board & 8.30 & 1 & 6.70 \\
& Misc. & 6.70 & 1 & 19.40 \\
& Total mass electronics & & & 4.04 \\
Propulsion & Coaxial shaft & 4.04 & 1 & 3.92 \\
& Blade & 0.98 & 4 & 1.00 \\
& Bearing & 0.25 & 4 & 0.76 \\
& Gear & 0.19 & 4 & 2.75 \\
& Stabilizer bar & 2.75 & 1 & 12.47 \\
& Total mass propulsion & & & 12.60 \\
Actuators & Motor & 6.30 & 2 & 7.66 \\
& Servo & 3.83 & 2 & 20.26 \\
& Total mass actuators & & & 12.70 \\
Battery & \multirow{2}{*}{12.70} & 1 & 95.84 \\
\hline & & & & \\
\hline & & & & \\
& & &
\end{tabular}

at $4 \%$, the thrust margin is already tiny. Thus, in order to add further components to the helicopter, ways need to be found to reduce the total mass, since a significant increase of the system's total thrust is not to be expected.

\subsection{Second Prototype}

With a largely defined rotor and propulsion system, the only way to reduce the helicopter's mass and consequently allow for addition of further components, is a tighter integration of all the components. The key to this is dual use of as many of components as possible, for instance by simultaneously using the necessary electronics as helicopter structure. Since the design goal for the muFly 2 prototype is a level of integration that is as high as possible, and since for this prototype all sensors and electronics can be produced to the specific need in terms of geometry, the design constraints differ from those of the muFly 1 prototype:

- Compulsory integration of the omnidirectional camera and the laser diodes as $x$ - $y$-position sensor.

- Compliance with a minimal distance of $90 \mathrm{~mm}$ between the laser plane and the camera focal center to achieve optimal resolution of the distance measurement.

- High structural stiffness to minimize displacement of laser diodes and camera with respect to each other, hence ensuring the quality of the high precision $x-y$ distance measurement. 
To meet these constraints, all electronics boards are dually used as structural parts. Horizontally and vertically placed Printed Circuit Boards (PCBs) intermesh, such that a three dimensional puzzle is established. This offers a lot of potential to save structural mass. Moreover, almost all electrical connections can be achieved by soldering the PCBs, making cables and connectors obsolete and offering further mass reduction potential for the electronics. The complete and assembled muFly 2 prototype is shown in Fig. 7. It can be seen that to a large extent the helicopter is built up from plate like structures that additionally serve as systems electronics. The only structural parts that do not serve a second purpose are a central plate to hold motors and servos, the landing gear, and bearing holders manufactured by rapid prototyping, which are necessary to hold the rotor shafts in position. These bearing holders serve as adaptors between the plate structures of the electronics and the cylindrical ball bearings. A complete overview of the disassembled prototype is shown in Fig. 8, where it becomes more obvious that the helicopter is mostly assembled from plate like parts. All components are placed in positions which correspond to their actual positions in the assembled versions. Most of the helicopter's structure is comprised of electronics, cables are only needed for the connections of the actuators, and in general the helicopter consists of a relatively small number of parts. Principally, the helicopter is mounted around a horizontal central structural plate, which holds the motors and the servos. The three vertical PCBs are pushed onto the plate and hold the bearing holders for the drive train in place from three directions. Other horizontal components held by the three vertical PCBs are the main processing board and the omnidirectional camera. On top and bottom, the vertical PCBs are held together by the laser diode PCB and the PCB for the ultrasonic range finder, respectively,

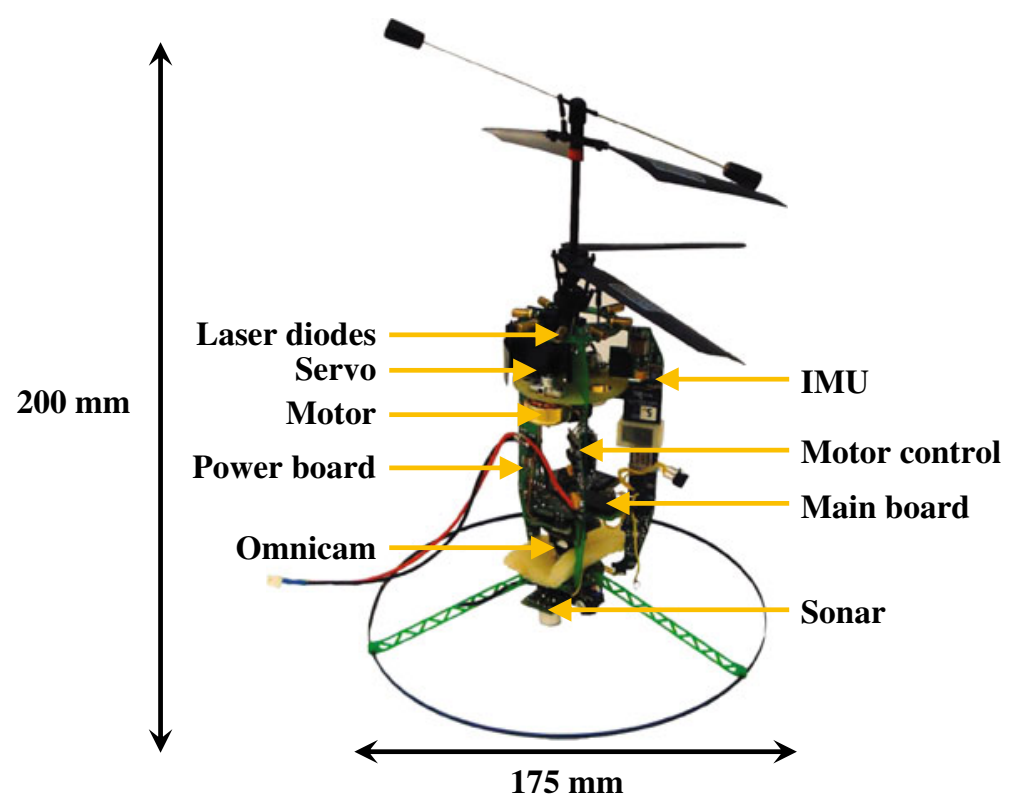

Fig. 7 Assembled muFly 2 prototype. It features high integration level thanks to dual use of the electronics as structural elements 
Fig. 8 Complete break up of the muFly 2 prototype. The design does not require cables or connectors, except for the battery

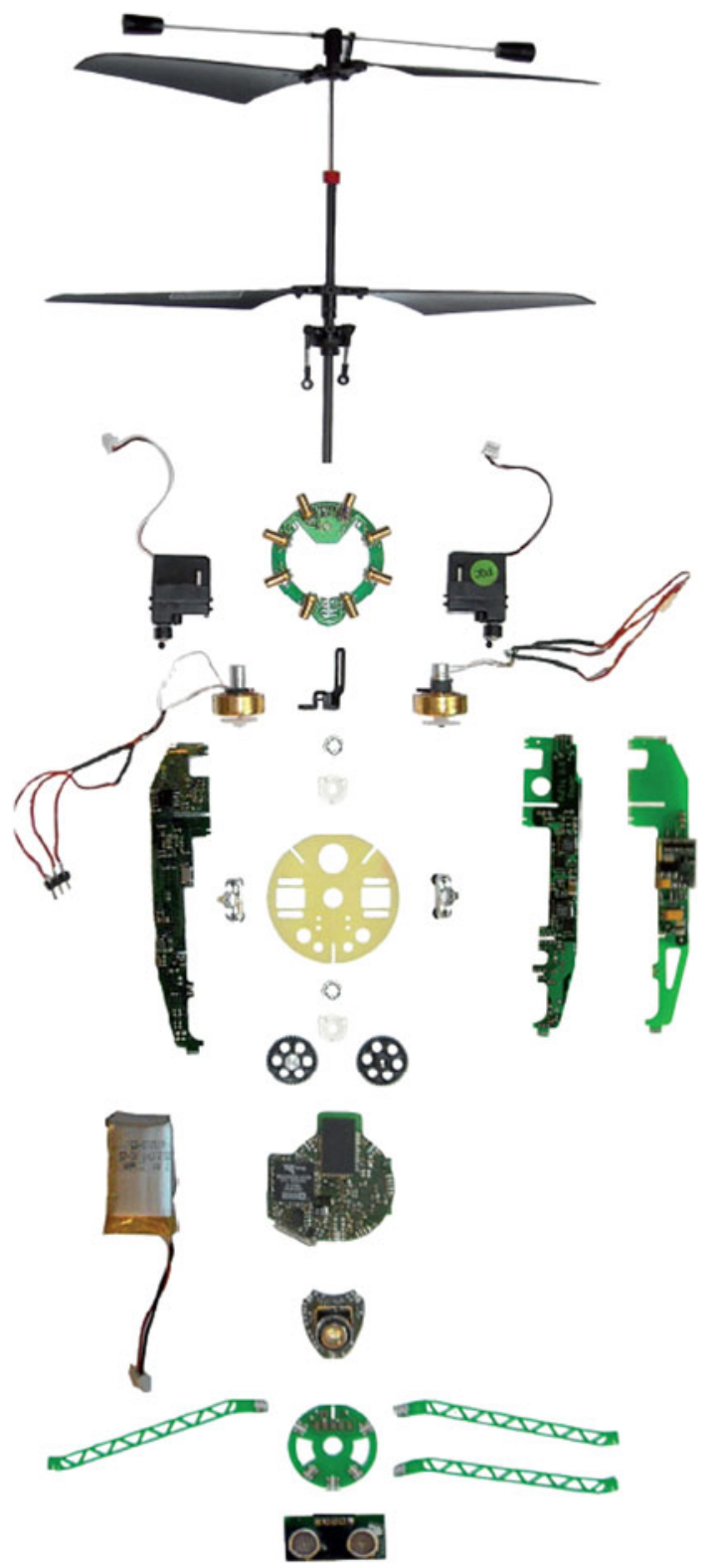

which are mounted as outer rings around the vertical PCBs. Hence, displacement of the vertical PCBs in the horizontal direction is prevented and a very stiff and stable structure is achieved. Of special interest, according to the design goals for this prototype, is the integration of a complete set of position sensors. These sensors are shown in Fig. 9 with the laser diodes (left), omnidirectional camera for detection of the laser points [11] (center) and ultrasonic range finder (right), the latter being the same as on the muFly 1 prototype. 

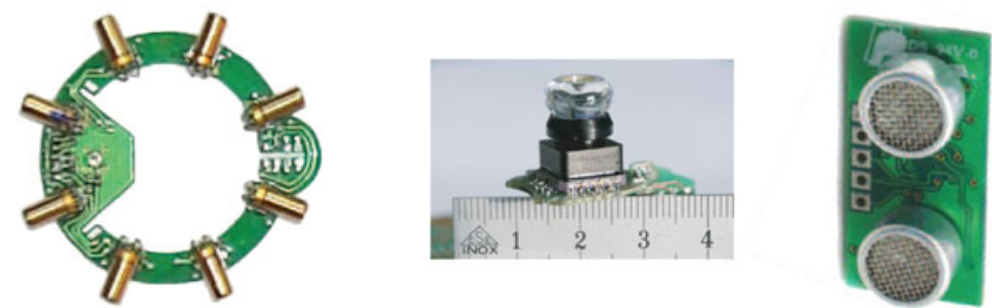

Fig. 9 Position sensors. Combined laser diodes (left) and omnidirectional camera (center), ultrasonic range finder (right)

The PCBs for the laser diodes and the omnidirectional camera are designed such that they can be integrated in the intermeshed three dimensional structure. A minimal distance of $90 \mathrm{~mm}$ must be obeyed in order to ensure optimal resolution of the optical measurement. Since the lowermost position on the helicopter is already reserved for the ultrasonic rangefinder to achieve a free field of view, the omnidirectional camera must be mounted higher than that. Therefore, with a height of $200 \mathrm{~mm}$, the helicopter appears to be higher than necessary. For this prototype of the muFly helicopter, a specialized BLDC outrunner motor has been developed by the project partner CEDRAT. This motor is an improved version of the BLDC motor LRK 13-4-15Y that is used for the muFly 1 prototype. The motor features silver wire wiring in an optimized arrangement for a higher motor efficiency at only slightly higher mass. This additional mass is mostly compensated by the fact that the motor includes its fixation, making additional motor holders as in the muFly 1 prototype obsolete. A complete overview over the mass distribution of the muFly 2 prototype is given in Table 2 . With a total mass of $80.31 \mathrm{~g}$ despite the added laser diodes and omnidirectional camera, the weight optimization of the second prototype becomes obvious. Moreover, the thrust margin of the helicopter is increased to almost $20 \%$. Another important observation is that the mass contribution of the helicopter's structure is very small, which is a result of the dual use of the system's electronic boards as structural parts. The major fraction of the second prototypes mass serves purposes that are actually relevant to the micro helicopter's autonomy.

\subsection{Prototype Comparison}

In this section, a quantitative comparison between the two muFly prototypes is made based on the mass data in Tables 1 and 2. The mass percentages of the main functional groups of prototype 1 are shown in Fig. 10 (left).

The major contribution to the total mass of $95.84 \mathrm{~g}$ is made by the actuators, i.e., motors and servos, which consume almost one quarter of the total mass. Other significant contributions come from the electronics and the structure. In general, it would be desirable to have high mass percentages of the sensors, the battery and to some extent also the electronics, because they can be considered to be useful payload either for autonomous flight or flight endurance. For the present result, their percentages are relatively low. The result for prototype 1 reflects its modular design, where easy exchangeability of standard components comes at the price of a high 
Table 2 Mass distribution of the muFly 2 prototype.

\begin{tabular}{llclc}
\hline & Component & $\begin{array}{l}\text { Comp. } \\
\text { mass }(\mathrm{g})\end{array}$ & $\begin{array}{l}\text { Quant. } \\
(-)\end{array}$ & $\begin{array}{l}\text { Total } \\
\text { mass }(\mathrm{g})\end{array}$ \\
\hline Structure & Central plate & 1.82 & 1 & 1.82 \\
& Landing gear & 1.34 & 1 & 1.34 \\
& Misc. & 0.63 & 1 & 0.63 \\
& Total mass structure & & & 3.79 \\
Sensors & IMU & 4.88 & 1 & 4.88 \\
& Laser diodes & 3.25 & 1 & 3.25 \\
& Camera & 3.57 & 1 & 3.57 \\
& Ultrasonic rangefinder & 3.75 & 1 & 3.75 \\
Electronics & Total mass sensors & & & 15.45 \\
& Motor contr. & 3.45 & 1 & 3.45 \\
& Power board & 3.92 & 1 & 3.92 \\
& Main board & 6.07 & 1 & 6.07 \\
& US PCB & 1.56 & 1 & 1.56 \\
Propulsion & Total mass electronics & & & 15.00 \\
& Coaxial shaft & 4.04 & 1 & 4.04 \\
& Blade & 0.98 & 4 & 3.92 \\
& Bearing & 0.20 & 2 & 0.40 \\
& Gear & 0.21 & 4 & 0.84 \\
& Stabilizer bar & 2.75 & 1 & 2.75 \\
& Total mass propulsion & & & 11.95 \\
Total mass & \multirow{2}{*}{6.88} & 2 & 13.76 \\
Actuators & Motor & 3.83 & 2 & 7.66 \\
& Servo & & & 21.42 \\
& Total mass actuators & & & 12.70 \\
& & 12.70 & 1 & 80.31 \\
\hline \multirow{5}{*}{ hattery } & & & \\
& & & & \\
& & &
\end{tabular}

structural mass fraction and low battery, sensors and electronics mass fractions. In Fig. 10 (right), a mass break up of the prototype 2 is shown. This prototype features a total mass of $80.31 \mathrm{~g}$, roughly $15 \mathrm{~g}$ lighter than the first prototype. The most obvious result from the mass break up is the reduction of the structural mass, which could be suppressed to a mere $5 \%$ of the total mass. This is a result of the design goal of a
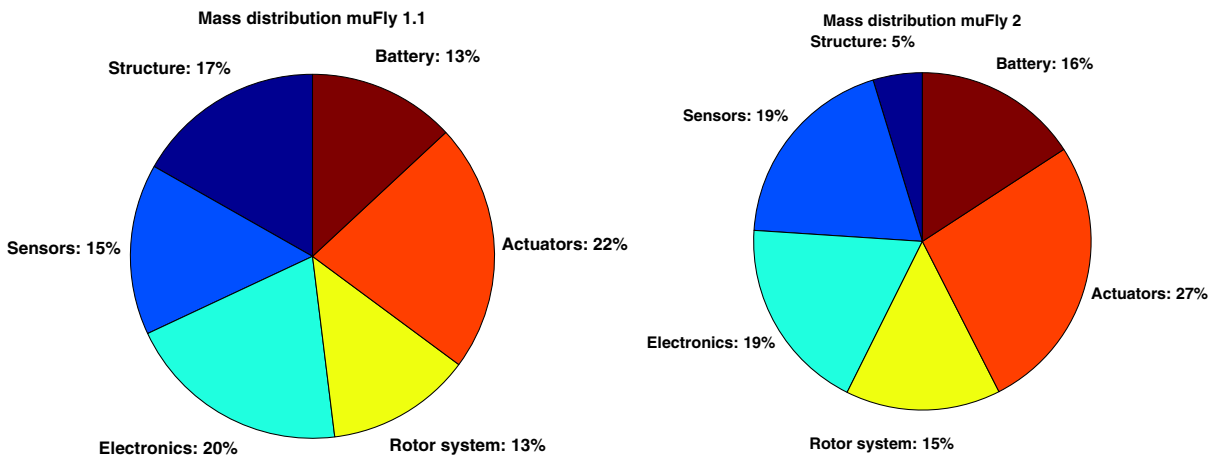

Fig. 10 Mass distribution over main functional groups for the prototype 1 at a total mass of $95.84 \mathrm{~g}$ (left), and prototype 2 at a total mass of $80.31 \mathrm{~g}($ right $)$ 

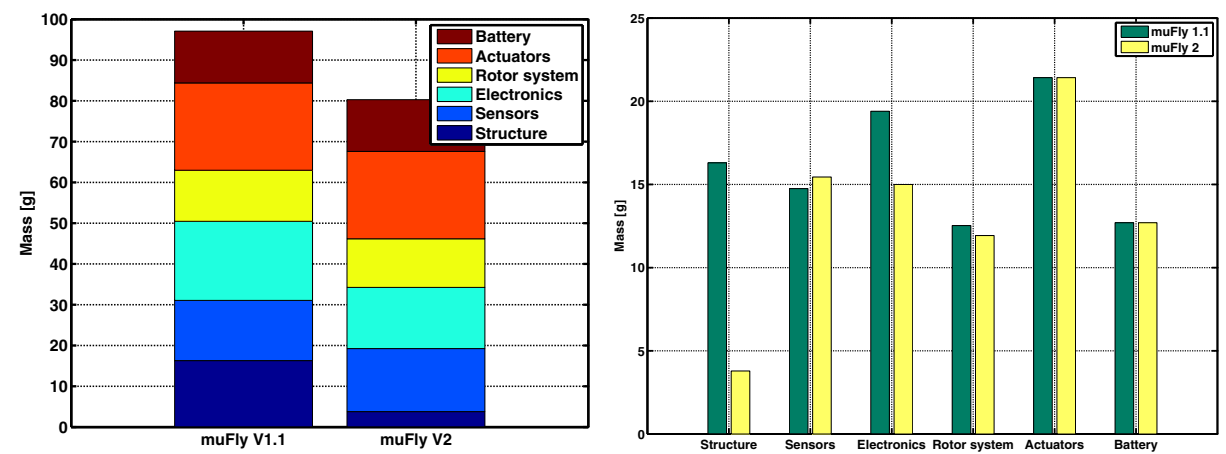

Fig. 11 Mass comparison and distribution over main functional groups for the two prototypes of muFly

strongly integrated helicopter that only uses specifically designed components, does not allow for easy exchange of components with standardized mechanical interfaces, and makes extensive use of dual purpose components, especially electronics, which also serve as structural parts. Another strong indicator for the success of dual use components is that the mass fraction of the electronics has not increased, in fact it is with $19 \%$ even slightly lower than for the prototype 1 . The raised percentage for the actuators is a result of the slightly increased mass of the optimized motors, while the total mass of the helicopter has decreased. Since the battery and the rotor system are exactly the same as on prototype 1 , their percentages have also slightly increased. The increase in the sensor percentage is obviously explained by the additional mass that is introduced by the position sensor consisting of the omnidirectional camera and the laser diodes. Moreover, this percentaged increase can be considered desirable. In total, the percentage of the useful payload of sensors, electronics and battery could be increased from $48 \%$ on prototype 1 to $54 \%$ on prototype 2 . Comparing the absolute mass values of the two prototypes, a similar result becomes visible. It is shown in Fig. 11 (left) for the complete helicopters, and in Fig. 11 (right) as a comparison of the functional groups.

Fig. 12 Prototypes muFly 1 (left) and muFly 2 (right) in flight
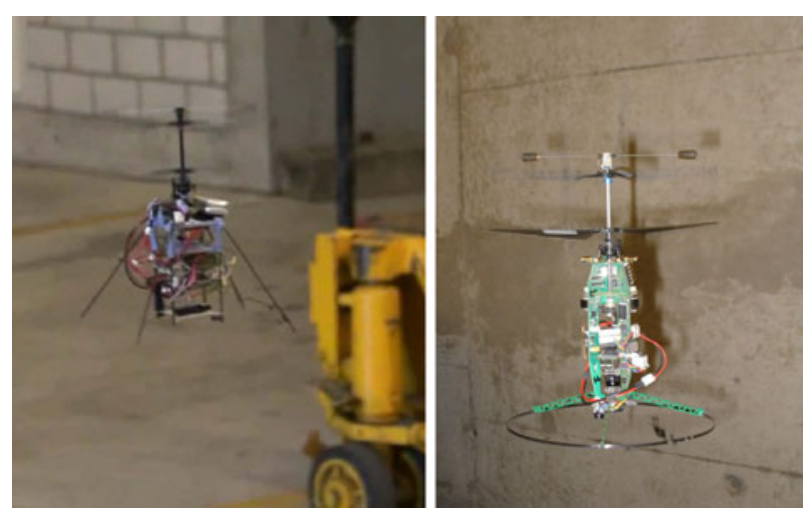
The significant mass reduction from 95.84 to $80.31 \mathrm{~g}$, which is a saving of $16 \%$, is mainly achieved by reduction of the structural and to some extent the electronics mass. Figure 12 shown the two prototypes.

\section{Partners' Contributions}

\subsection{Micro Laser-Omnicam}

For collision avoidance an omniview camera has been developed. The system has been equipped with several pointing lasers to use it for multidirectional triangulation. Figure 13 shows the principle set-up. For this part of the project, the challenge has been the requirements concerning the power consumption, the image processing time, the size and the weight of the omniview camera: how can be the system miniaturized, to make it so small and lightweight that it can be used as a navigation aid for an autonomous flying micro-robot. Omniview cameras have a horizontal field of view of $360^{\circ}$. They can be realized with several cameras or rotating cameras. For compact systems, wide-angle lenses or lenses combined with cone-like mirrors are used (catadioptrical lens). Wide-angle lenses have a strong predominance of the sky in their image. In a catadioptrical omnicam system, usually the mirror is a separate component, and the overall size of the system is in the order of several tens of centimeters, which is unpractical for a flying micro-robot. The panoramic image of such a catadioptrical camera is captured in one frame and in polar coordinates, i.e., horizontal object structures in the panoramic scene appear on the sensor as a circle, vertical structures as radial stripes. The unwrapping of such a panoramic image captured by a sensor with Cartesian coordinates is quite processor time consuming. Here, we report on the miniaturization of such a catadioptrical system: In a novel optical design the mirror has been integrated into the lens system of the camera. The image sensor of the camera has been realized with a polar pixel

Fig. 13 Principle set-up of the multidirectional triangulation system with a miniaturized omniview camera and eight lasers

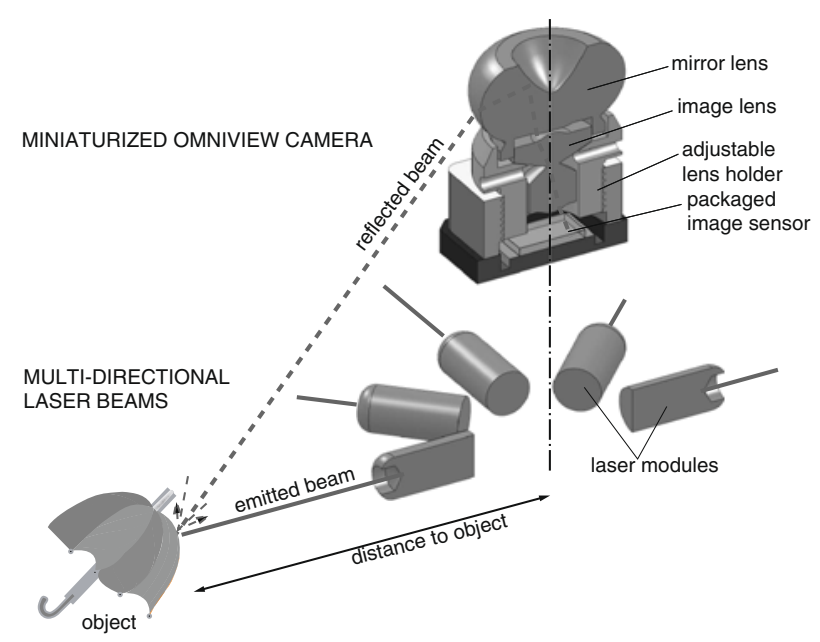


field to avoid computation effort for the enrollment of the panoramic image; it is a low-power CMOS sensor, and, due to the innovative technology, it provides a high dynamic range.

\subsubsection{Preliminary Prototype Development}

The requirements of the triangulation system can be split in the requirements for the sensor, for the optical system, for the camera and for the overall triangulation system, see Table 3.

\subsubsection{Image Sensor Development}

Depending on the environmental conditions, there can occur very bright and very dim areas in one picture. In case of a pixel with a linear response, the bright areas are over-exposed or the dim areas are under-exposed. Here, CSEM's PROGLOG ${ }^{\text {TM }}$ technology is used: Below a certain amount of light, the response of the photodiode is linear whereas above this programmable threshold, the photodiode response is logarithmic; thereby an over-exposure is avoided [11]. For the effortless transformation of the cylindrical image into Cartesian coordinates, a polar pixel field layout with 64 concentric circles (rows) and 128 radials (columns) has been designed. The radial height of each pixel is $30 \mu \mathrm{m}$, and the diameter of the pixel field is $4 \mathrm{~mm}$. The width of the pixels is increasing proportionally with the radius. Since the number of pixels per circle is constant, the unwrapped image resolution is a constant, too. The light response of each pixel has been adapted, since a similar sensitivity is required for all pixels, despite their spatially variant width in the polar-radial geometry. This is achieved by designing pixels with different size and shape but keeping an identical ratio of effective capacitance to geometrical fill factor (fF/um2). Figure 14 shows a picture of the polar pixel field sensor in its package.

Table 3 Requirements for the multidirectional triangulation system

\begin{tabular}{|c|c|c|}
\hline Parameter & Value & Unit \\
\hline \multicolumn{3}{|l|}{ Sensor } \\
\hline Geometry & Polar pixel field & \\
\hline Power consumption & $<1$ & $\mathrm{~W}$ \\
\hline Dynamic range & no blooming by laser & $\mathrm{dB}$ \\
\hline Output precision & 10 & bit \\
\hline Frame rate & 40 (programmable) & fps \\
\hline \multicolumn{3}{|l|}{ Optics } \\
\hline Spot size & $<$ pixel size & \\
\hline Volume (optics) & 2 & $\mathrm{~cm}^{3}$ \\
\hline \multicolumn{3}{|l|}{ Camera } \\
\hline Mass (camera) & $<5$ & $\mathrm{~g}$ \\
\hline Volume (camera) & $<5$ & $\mathrm{~cm}^{3}$ \\
\hline \multicolumn{3}{|l|}{ Triangulation System } \\
\hline $\begin{array}{l}\text { Weight (triangulation } \\
\text { system incl. laser PCB) }\end{array}$ & $<10$ & $\mathrm{~g}$ \\
\hline Resolution & 0.1 & $\mathrm{~m}$ \\
\hline Distance range & $0.3-3$ & $\mathrm{~m}$ \\
\hline
\end{tabular}


Fig. 14 Picture of the image sensor. One can clearly see the circular shape of the light-sensitive part. The package is about $11 \times 11 \mathrm{~mm}$ in size

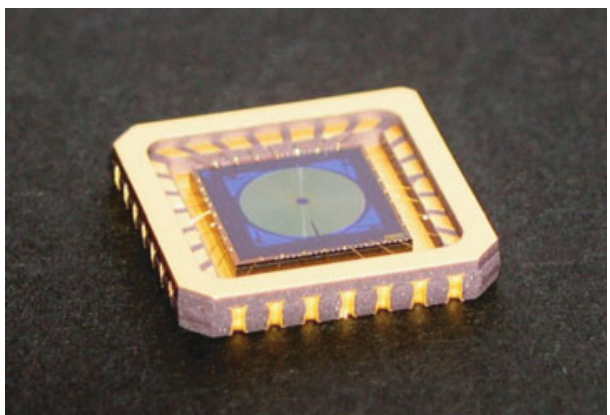

\subsubsection{Optic Design}

In common catadioptric systems for omnidirectional cameras, the mirror is a separated component, its design is independent of the imaging lens of the camera. Here, in a first miniaturized prototype, the mirror has been integrated into the lens system. Such a mirror lens is a catadioptrical lens with a toroidal input facet, a mirror and an output facet [12]. The preliminary prototype has been designed with a field of view of FoV $=-35^{\circ}$ to $+10^{\circ}$ in the vertical direction. Figure 13 shows the principle optical set-up. Imaging lens and mirror lens are aspheres. They have been fabricated as prototypes by ultra precision diamond turning (Eschenbach Optik $\mathrm{GmbH})$. The metal coating has been evaporated in the hollow cone. A lens holder has been designed which allows to adjust the focal length of the catadioptric lens to compensate fabrication tolerances.

\subsubsection{Characterization of the Preliminary Prototype}

The camera has been mounted and the basic functionality tested. Especially the dynamic range of the sensor and the image quality of the optical system were of interest. The sensor has been placed in an environment with very bright light sources. By adjusting the voltage for the logarithmic output threshold, blooming effects have been avoided as shown in Fig. 15.

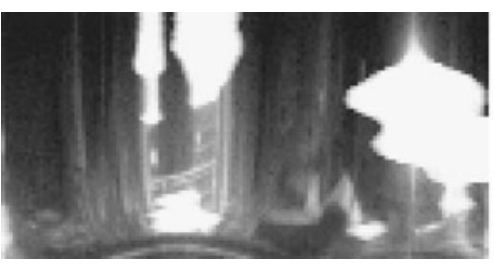

(a)

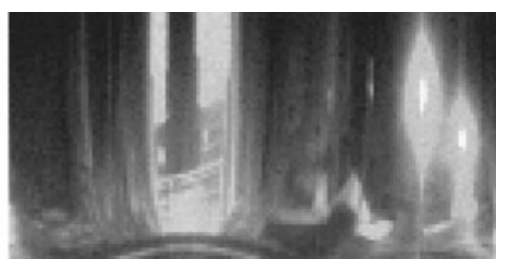

(b)

Fig. 15 Demonstration of the PROGLOG pixel technology. The images are unwrapped panoramic pictures, which show a window with a black panel in the center and two light bulbs at the right. They were captured with the presented camera without (a) and with (b) the PROGLOG functionality 


\subsection{Final Prototype}

\subsubsection{Optical System Design for Triangulation}

The final prototype is based on the image sensor as described in the previous section. The final optical system design has been improved further for triangulation: Usually, in a non distorting system, the reflected laser spot is impinging on the pixel-field at a radius, which is inversely proportional to the distance of the object. Here a design dedicated for triangulation has been realized, where the vertical (radial) image axis has been distorted to increase the optical resolution of the laser beam position for distant objects. The total vertical field of view has been decreased to $\mathrm{FoV}=-20^{\circ}$ to $-1^{\circ}$, the distance between the lasers and the camera is $93 \mathrm{~mm}$. If one excludes interpolation methods, a measure of the distance resolution is the minimum detectable distance increment, which is indicated by the change of the spot position on the pixel field by one pixel. In Fig. 16 this pixel resolution is plotted for different designs: for a conventional design, for the preliminary prototype design, for the ideal distorted system and for the final prototype. As can be derived from the Fig. 16, the resolution in a triangulation application is improved by a factor of three by the design of the final optical system.

\subsubsection{Characterization of the Triangulation System}

The final system has been assembled with eight lasers in a distance of $93 \mathrm{~mm}$ to the optical horizontal plane of the omniview camera. Each laser diode is emitting $5 \mathrm{~mW}$ optical power at $635 \mathrm{~nm}$ (APCD-635-07-05-A, Arima Lasers). In a dark room, the prototype has been installed in front of a moving target covered with white paper. The measurement has excluded any interpolation algorithms and thus is comparable only to a 1 bit output resolution per pixel (see Fig. 17). Resolution values are listed in Table 4, which gives an overview of the achieved results of the final prototype.

Fig. 16 Pixel resolution excluding interpolation for various optical designs and for the final prototype design

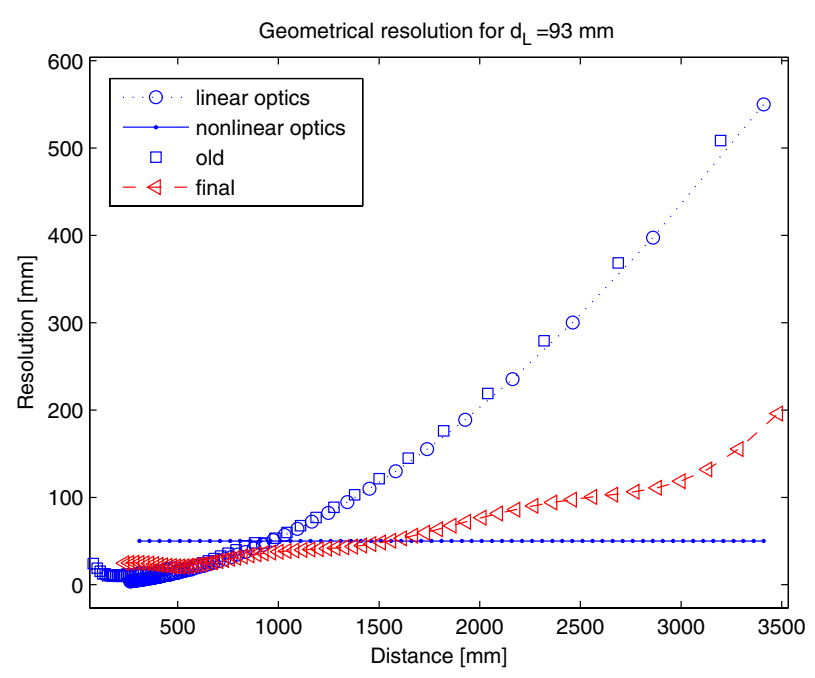




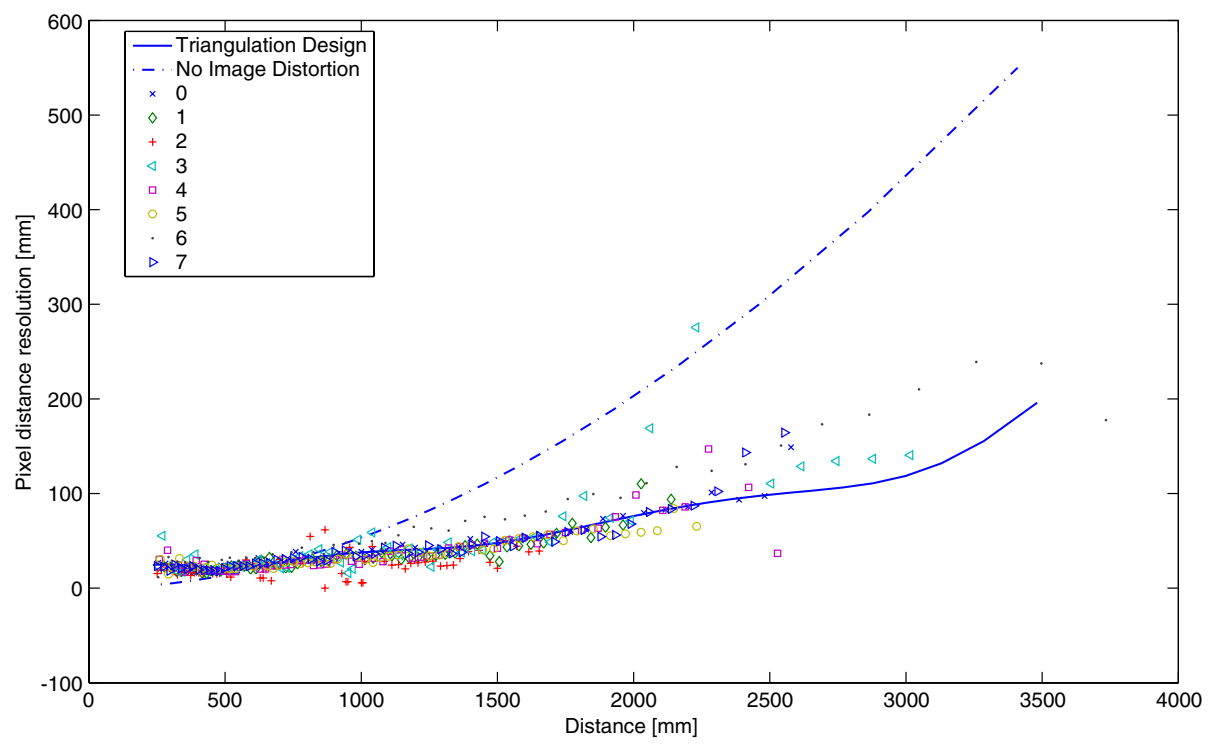

Fig. 17 Multi-directional triangulation measurements with eight mounted lasers (possibility for up to 128 theoretical). Each laser is emitting $5 \mathrm{~mW}$ optical power

\subsection{Inertial Measurement Unit}

The Inertial Measurement Unit (IMU) of muFly is a special design made by the company Xsens (http://www.xsens.com/). In fact, the final version of the helicopter

Table 4 Achieved results of the final prototype

\begin{tabular}{lll}
\hline Parameter & Value & Unit \\
\hline Sensor & & \\
Geometry & 64 circles $\times 128$ radials & - \\
Power consumption & 0.006 & $\mathrm{~W}$ \\
Dynamic range & 140 & $\mathrm{~dB}$ \\
Output precision & 10 & $\mathrm{bit}$ \\
$\quad$ Frame rate & 40 (programmable) & $\mathrm{fps}$ \\
Optics & & \\
Spot size radius & $0.015(\mathrm{RMS}$, inner circle) & $\mathrm{mm}^{3}$ \\
$\quad$ Volume (optics) & $16.5 \times 10.5 \times 10.5$ & $\mathrm{~mm}^{3}$ \\
Camera & 3 & \\
Mass (camera) & $20 \times 23 \times 19$ & $\mathrm{~g}$ \\
$\quad$ Volume (camera) & & $\mathrm{mm}$ \\
Triangulation System & 8 & $\mathrm{~g}$ \\
$\quad$ Weight (triangulation & & \\
$\quad$ system incl. laser PCB) & & $\mathrm{mm}$ \\
Distance laser to optical axis & 92 & $\mathrm{~mm}$ \\
Resolution & & $\mathrm{mm}$ \\
@1m distance & 50 & $\mathrm{~mm}$ \\
@2m distance & 100 & $\mathrm{~m}$ \\
@3m distance & 200 & \\
Distance range & $0.25-3.5$ &
\end{tabular}


Fig. 18 The IMU of muFly. The elongated shape imposed strong constraints on the analog chain design

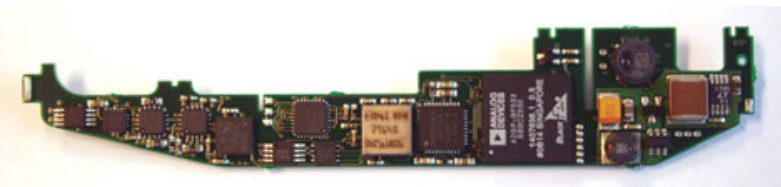

makes a double use of the electronics as structural elements. The IMU is one of the three arms of the structure. This imposes an elongated form-factor for the PCB (see Fig. 18) and puts an additional constraint, especially on the analog-chain design. Xsens made a whole study about the best raw sensors to be used on muFly. The IMU has one ADXRS610 and one IDG300 gyroscopes, one LIS accelerometer from ST, an HMC magnetometer and an SCP pressure sensor. The data fusion is realized through the proprietary Kalman filter from Xsens.

\subsection{Micro Actuation}

The role of the company CEDRAT technology during muFly was to design micro actuators with high power to weight ratio. The first muFly prototype had to be equipped with commercially available micro-motors (Mighty Midget 13/4/15), giving more time for CEDRAT to design muFly-optimized actuators. The choice of outrunner BLDC motors technology was quite obvious thanks to the higher torque capability compared to other technologies. Table 5 compares six different BLDC micro motors, among the best available on the market in 2006. CEDRAT was also in charge of developing very small linear actuators, to be coupled with a micro swashplate for the helicopter steering purpose. Figure 19 shows the lower rotor of muFly prototype V1.0, to which are connected the micro swashplate and the piezoelectric linear-actuators (DTTuXS). The total mass of these actuators was about $6 \mathrm{~g}$, including drive electronics (Cau10). The stroke was the main limitation, since it was hardly reaching $6^{\circ}$ (total stroke). However, the bandwidth of $30 \mathrm{~Hz}$ and the precision of a couple of micrometers were excellent. The combination DTTuXS and Cau10 is now a standard product at CEDRAT. The most important contribution of CEDRAT in muFly was for sure the high-performance BLDC motors (and their controllers), built from scratch and optimized for muFly, while having the same mechanical and electrical interfaces as the off-the-shelf motor. CEDRAT developed

Table 5 Micro-motors evaluation chart

\begin{tabular}{lllllll}
\hline & Maxon & Faulhaber & Mighty M. & Mighty M. & LRK & MiniDisc \\
& EC6 & 06 series & $10 / 3 / 26$ & $10 / 3 / 26 \mathrm{D}$ & $195-03$ & BL \\
\hline Rotor config. & In & In & Out & Out & Out & Out \\
Pwr/mass (W/g) & $0.43 @ 6 \mathrm{~V}$ & $0.62 @ 6 \mathrm{~V}$ & $0.36 @ 3.5 \mathrm{~V}$ & $0.66 @ 3.5 \mathrm{~V}$ & $0.9 @ 7 \mathrm{~V}$ & $0.41 @ 7 \mathrm{~V}$ \\
Trq/mass (N.m/Kg) & 0.09 & 0.148 & - & - & - & - \\
Weight (g) & 2.8 & 2.5 & 3.5 & 3.5 & 12 & 7.9 \\
Control & Hall & Hall & - & - & - & - \\
Max. efficiency & 50 & 57 & 52 & 54 & 72 & 39 \\
\hline
\end{tabular}



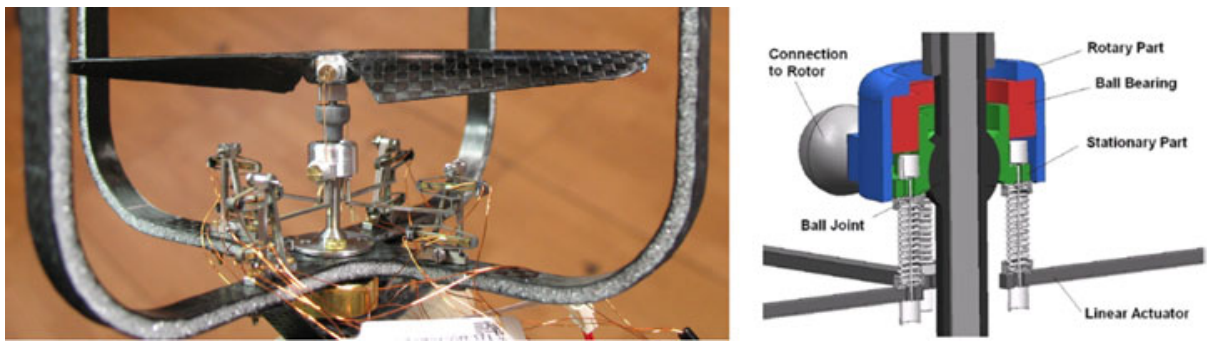

Fig. 19 Left The lower rotor of muFly prototype V1.0, with its micro swashplate and piezo-electric linear-actuators. Right Basic elements of the micro swashplate

a fabrication process including a motor winding method and an assembly procedure. In order to get the best performance, high grade curved magnets were used. The curved shape ensures a constant air-gap, which enhances slightly the efficiency by $2-4 \%$. Moreover, copper wires were replaced with silver ones which increases the efficiency by $1-2 \%$, thanks to the better conductivity of Silver. CEDRAT used also thinner laminated sheets for the rotor, which provides in theory $4 \%$ more efficiency (see Fig. 20).

\subsection{Power Source}

After the first year of muFly project, it was clear that a fuel-cell which would fulfill the requirements of muFly in term of power supply would be too heavy, almost heavier than the target mass of the total system. Thus, a Lithium Polymer battery was still the best solution at that size. Nevertheless, it was decided to continue the development of the low-weight, high power fuel-cell stack for many other application which would go beyond micro helicopters. A light weight fuel cell stack with passive thermal management for a $12 \mathrm{~W}$ hydrogen Proton Exchange Membrane (PEM) fuel cell was developed. This was achieved with help of a Bi-cell design (see Fig. 21) were
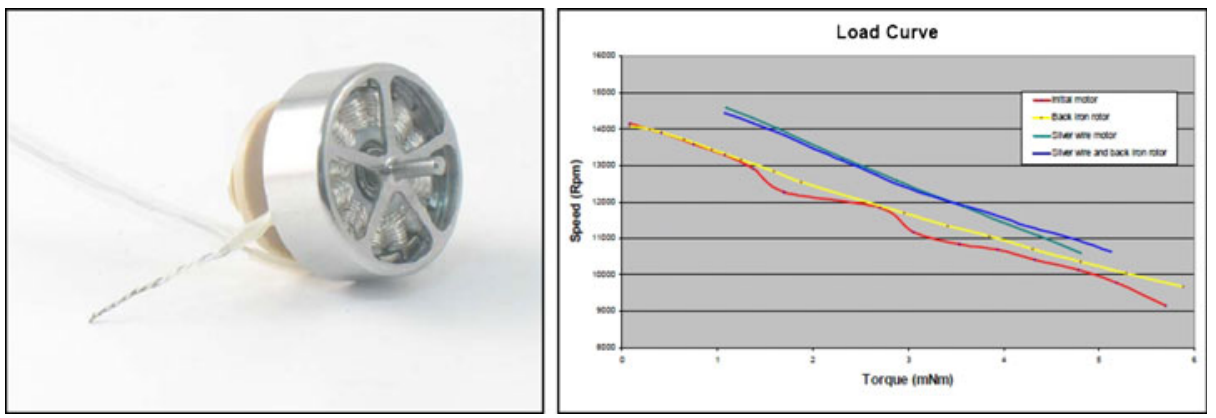

Fig. 20 Left The new enhanced motor $(6.25 \mathrm{~g})$, designed and produced by CEDRAT. Right The load curve of the new motor compared with the off-the-shelf one 

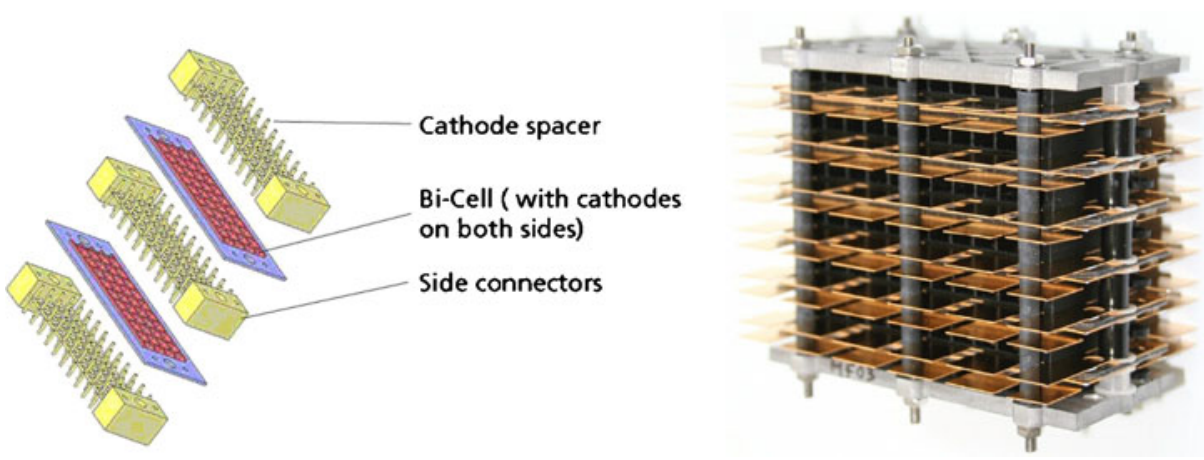

Fig. 21 Left Principle of fuel cell stack. Right 12 cell fuel cell stack

the fuel cells are separated with air spacers which allow to use the air flow of the rotors for cooling and cathode supply. The construction based on thin wall polymer flow channels and aluminum metal foil current collectors resulted in very low weight. The aluminum current collectors protrude from the active are of the fuel cells to act as additional air cooling area as can be seen in Fig. 21. The design resulted in a stack weight of $90 \mathrm{~g}$. Further weight reductions were achieved by replacing the metal screws with carbon fibers. The current voltage characteristic is shown in Fig. 22 at the bottom. At $25^{\circ} \mathrm{C}$ ambient temperature a power of $15 \mathrm{~W}$ can be produced over long periods of time. The system was demonstrated with a quadrotor helicopter were the fuel-cell and the reactor were fixed in a defined distance below the rotors. Sufficient cooling and stable power delivery was demonstrated during almost 10-min flight time (see Fig. 23). A power density between 60 and $160 \mathrm{~W} / \mathrm{kg}$ can be achieved for the complete system depending on the size of the fuel cell cartridge.

Fig. 22 Current voltage characteristic of the fuel cell stack

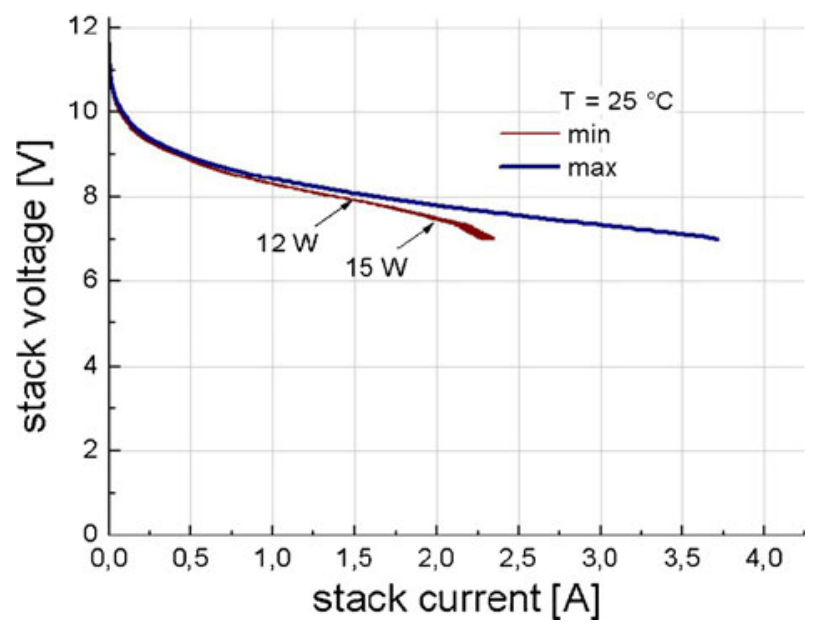



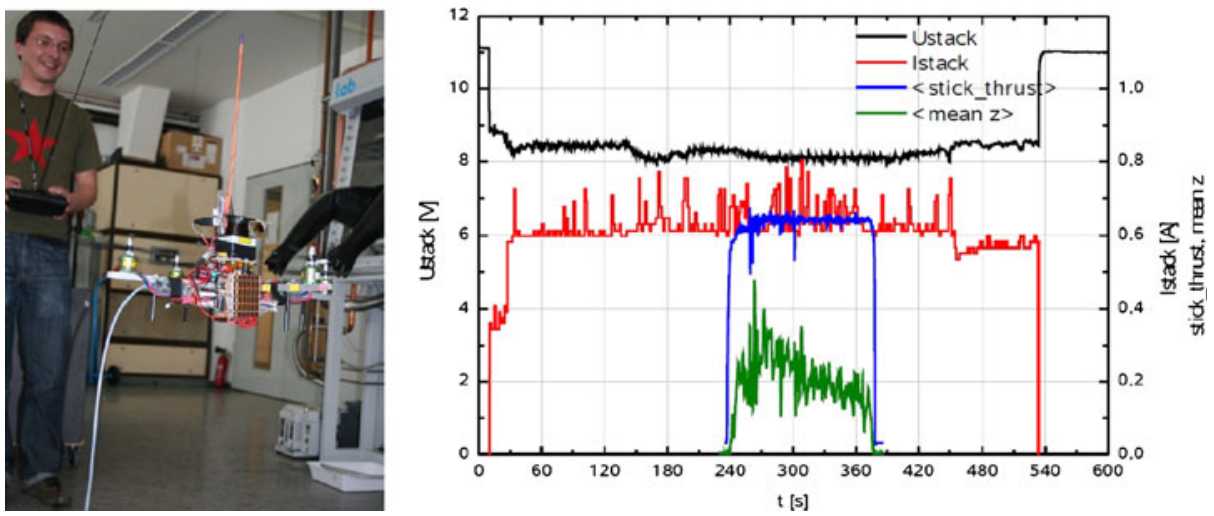

Fig. 23 Left Test of the power generation during flight. Measured data from fuel cell and quadrotor. Booting the CPU the first $40 \mathrm{~s}$, flying in between second 240-380. Here, <mean $z>$ is the current height estimate of the quadrotor, whereas $<$ stick_thrust $>$ is the thrust command send via the remote control (indicating the whole cooling period)

\section{Navigation}

During the design and construction of the different muFly prototypes, the navigation algorithms were being developed on a bigger flying platform, a quadrotor. At the end of the project it was not possible to transfer all algorithms on the muFly helicopter due to sensory setup and the lack of processing power. This section presents the setup and the algorithms for estimating the pose of a flying vehicle within a known environment and for online simultaneous localization and mapping. For validating the algorithms we use a modified Mikrokopter (http://www.mikrokopter.de/) quadrotor illustrated in Fig. 24. We equipped the quadrotor with a Hokuyo URG laser range scanner and a low-cost MTi XSens IMU. The laser range finder is able to measure distances up to $5.6 \mathrm{~m}$ with an angular resolution of approximately $0.35^{\circ}$. To measure the altitude of the vehicle with respect to the ground we deflect several laser beams towards the ground with a mirror. The remaining beams are used for 2D localization and SLAM. The XSens provides orientation angles with a dynamic accuracy of
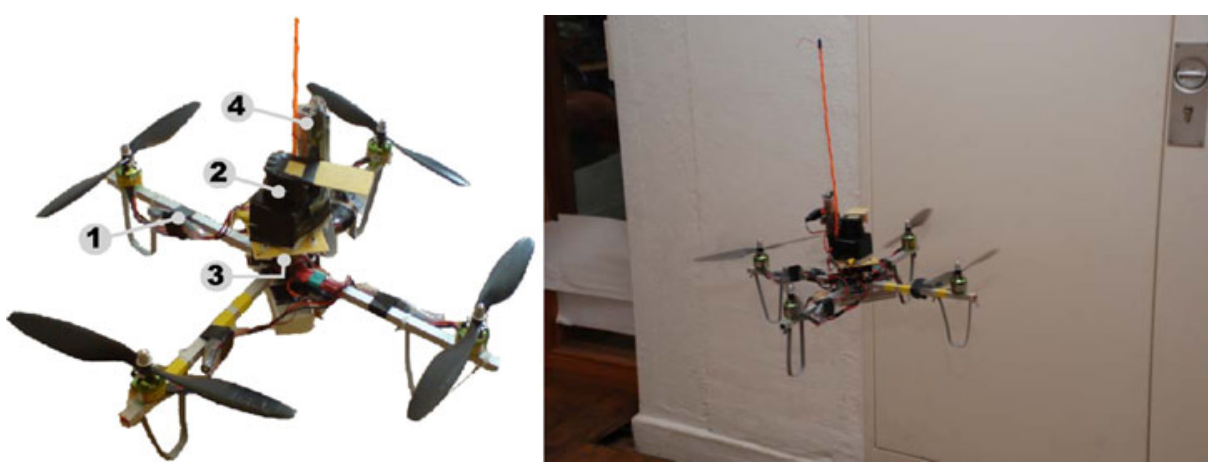

Fig. 24 Our quadrotor (left): 1 Mikrokopter platform, 2 Hokuyo laser range finder, 3 XSens IMU, 4 Gumstix computer. Right Our system during a mission 
$2^{\circ}$. The on-board computation is performed by a PXA-based embedded computer (Gumstix-verdex) running at $600 \mathrm{Mhz}$. This combination of laser-scanner and IMU allows us to simplify the state estimation problem by reducing the state space from 6 to 4 dimensions, since accurate roll and pitch angles are available from the IMU. Partitioning the remaining 4DOF into $(x, y, \theta)$ and $z$, makes it possible to use the broad range of existing algorithms for $2 \mathrm{D}(x, y, \theta)$ wheeled mobile robot localization and SLAM.

\subsection{Localization}

We apply a particle filter [13] algorithm to estimate the current pose of the vehicle. In contrast to other filtering techniques, like Kalman Filters, particles filters are able to deal with highly non-linear systems and can approximate arbitrarily complex density functions. This property includes multi-modal pose estimation as well as global localization, i.e., when the starting pose of the vehicle is not known in advance. The key idea of Monte Carlo localization it to estimate the possible robot locations using a sample-based representation. Formally, the task consists in estimating the posterior $p\left(\mathbf{x}_{t} \mid \mathbf{z}_{1: t}, \mathbf{u}_{1: t}\right)$ of the current robot pose $\mathbf{x}_{t}$ given the a known map of the environment, the odometry measurements $\mathbf{u}_{1: t}=\left\langle\mathbf{u}_{1}, \ldots, \mathbf{u}_{t}\right\rangle$ and the observations $\mathbf{z}_{1: t}=\left\langle\mathbf{z}_{1}, \ldots, \mathbf{z}_{t}\right\rangle$ made so far. In the particle filter framework, the probability distribution about the pose of the robot at time step $t$ is represented by a set of weighted samples $\left\{\mathbf{x}_{t}^{[j]}\right\}$. The robustness and efficiency of this procedure strongly depends on the proposal distribution that is used to sample the new state hypotheses in the selection step. Since our flying vehicle does not provide reliable odometry measurements, we apply an incremental scan-matching procedure to estimate the inter-frame motion of the vehicle.The localization of one experiment performed at a flying height of $50 \mathrm{~cm}$ with 5,000 particles for global localization is depicted in Fig. 25. The top-left (a) image shows the initial situation in which the current pose
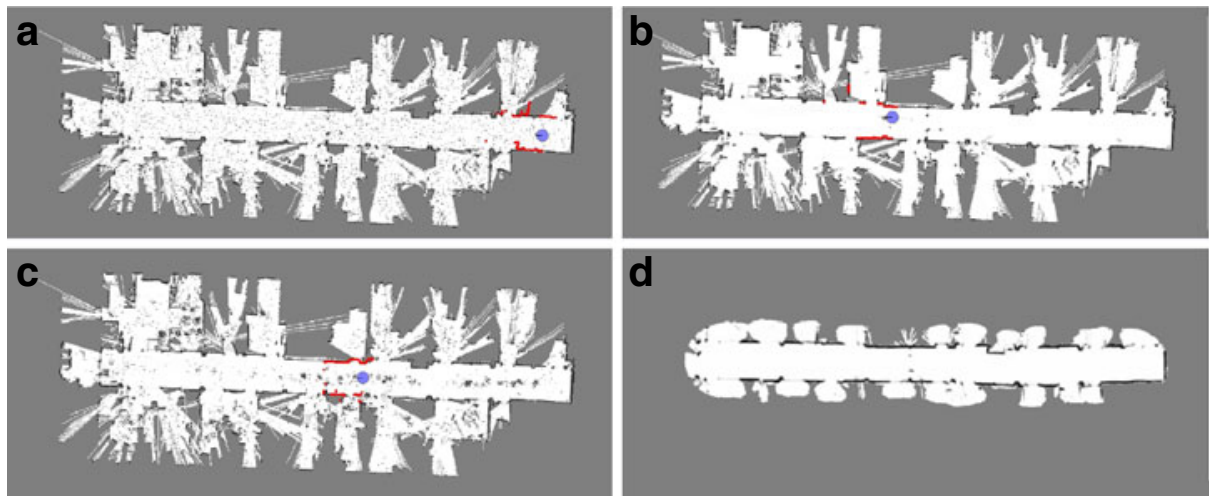

Fig. 25 Global localization of our quadrotor (a-c). Top Initial situation, with uniformally drawn random poses. Middle After about $1 \mathrm{~m}$ of flight, the particles start to focus on the true pose. Bottom After approximately $5 \mathrm{~m}$ of flight the particle set has focused around the true pose of the helicopter. The blue circle highlights the current best estimate of the particle filter. The quadrotor was able to autonomously maintain its height of $50 \mathrm{~cm}$ during this experiment. A self-build map of our office environment utilizing our approach and using the quadrotor is shown in $\mathbf{d}$ 
of the quadrotor is unknown. After few iterations (i.e., after about $1 \mathrm{~m}$ of flight) the localization algorithm starts to focus on relatively few possible poses only (b). After about $5 \mathrm{~m}$ of flight, the particles are highly focused around the true pose of the helicopter (see (c) image of Fig. 25). Note that we highlighted the maximum a posteriori pose estimate in the three snapshots.

\subsection{Simultaneous Localization and Mapping}

Our mapping system addresses the SLAM problem by its graph based formulation. A node of the graph represents a 3 DoF pose of the vehicle and an edge between two nodes models a spatial constraint between them. These spatial constraints arise either from overlapping observations or from odometry measurements. In our case the edges are labeled with the relative motion between two nodes which determine the best overlap between the scans acquired at the locations of the nodes. To compute the spatial configuration of the nodes which best satisfy the constraints encoded in the edges of the graph, we use an online variant of a stochastic gradient optimization approach [14]. Performing this optimization on the fly allows us to reduce the uncertainty in the pose estimate of the robot whenever constraints between nonsequential nodes are added. The result of a typical run is shown in Fig. 25d.

\section{Conclusion}

This paper presented the results achieved during the EU project muFly. It discussed the approach based on designing first, a series of test-benches in order to understand the problematic, before designing the flying vehicle. Based on this approach, two prototypes were designed and presented in this paper. The final one weighs about $80 \mathrm{~g}$ for $17 \mathrm{~cm}$ total span. The tiny laser omnicam that was presented in this paper, represents one of the major developments in muFly project. Coupled with the laser module, it represents a $8 \mathrm{~g}, 360^{\circ}$, solid-state range finding solution running at $30 \mathrm{~Hz}$. The paper also presented the $6.5 \mathrm{~g}$ BLDC micro motor with silver wire and thin lamination that achieved about $50 \%$ efficiency. The $12 \mathrm{~W}$ patented fuelcell solution was also presented. It was not used on the final muFly, but represents nevertheless a interesting development useful for different applications. Finally, the paper showed that the navigation algorithms developed during muFly led to the highly autonomous quadrotor. In summary, one can say that muFly project generated several developments at the system (helicopter) and sub-systems levels, with application that go beyond the field of robotics.

Acknowledgements This work has been supported by the EC under contract number FP6-IST034120 Micro/Nano based Systems. ETHZ, the leading partner of muFly would like to thank the EC for the funding and the valuable feedback. We want also to thank all the persons who participated directly or indirectly to this exciting project. Special thank to Markus Bühler and Dario Fenner for the numerous mechanical parts that were not always easy to manufacture due to their size. Special tank also to Janosch Nikolic and Thomas Baumgartner for their precious help on the electronics design. We are specially grateful to Stephan Weiss, without his help, the laser-module would not have been possible. We would also like to thank Giorgio Grisetti and Bastian Steder for their enormous help within the project. 


\section{References}

1. Pines, D., Bohorquez, F.: Challenges facing future micro air vehicle development. AIAA J. Aircr. 43(2), 290-305 (2006)

2. Kroo, I., et al.: The mesicopter: a miniature rotorcraft concept phase 2 Interim Report (2000)

3. University of Florida GATOR: http://www.mae.ufl.edu/mav/

4. Schafroth, D., Bouabdallah, S., Bermes, C., Siegwart, R.: From the test benches to the first prototype of the muFly micro helicopter. J. Intell. Robot. Syst. 54(1-3), 245-260 (2008)

5. Bermes, C.: Dynamic modeling and design of coaxial micro helicopters. Ph.D. Thesis, ETH Zürich (2010)

6. Morris, M.v.N.J.C., Bendotti, P.: Identification and control of a model helicopter in hover. In: Proceedings of the American Control Conferences, Baltimore, Maryland. American Automatic Control Council (1994)

7. Lidstone, C.: The Gimballed helicopter testbed: design, build, and validation. Tech. Rep., System Control Group at University of Toronto (2003)

8. Weilenmann, M.F., Geering, H.P. (1994) Test bench for rotorcraft hover control. J. Guid. Control Dyn. 17(4) (1994)

9. Oesterlin, W.: Ein flugfähig eingespannter modellhubschrauber zur untersuchung des menschlichen reglerverhaltens. In: Zeitschrift für Flugwissenschaften und Weltraumforschung (1979)

10. Schafroth, D.: Aerodynamics, modeling and control of an autonomous micro helicopter. Ph.D. Thesis, ETH Zürich (2010)

11. Ferrat, P., et al.: Ultra-miniature omni-directional camera for an autonomous flying micro-robot. In: SPIE Photonics Europe Conference, Strasbourg, France (2008)

12. Gimkiewicz, C., et al.: Ultra-miniature catadioptrical system for an omnidirectional camera. In: SPIE Photonics Europe Conference, Strasbourg, France (2008)

13. Thrun, S., Burgard, W., Fox, D.: Probabilistic Robotics. MIT Press (2005)

14. Grisetti, G., Lodi Rizzini, D., Stachniss, C., Olson, E., Burgard, W.: Online constraint network optimization for efficient maximum likelihood mapping. In: Proc. of the IEEE Int. Conf. on Robotics \& Automation (ICRA), Pasadena, CA, USA (2008) 